\title{
Michigan Infrared Combiner (MIRC): commissioning results at the CHARA Array
}

John D. Monnier, Ettore Pedretti, Nathalie Thureau, Jean-Philippe Berger, Rafael Millan-Gabet, et al.

John D. Monnier, Ettore Pedretti, Nathalie Thureau, Jean-Philippe Berger,

Rafael Millan-Gabet, Theo ten Brummelaar, Harold McAlister, Judit

Sturmann, Lazlo Sturmann, Phil Muirhead, Ajay Tannirkulam, Scott

Webster, Ming Zhao, "Michigan Infrared Combiner (MIRC): commissioning results at the CHARA Array," Proc. SPIE 6268, Advances in Stellar

Interferometry, 62681P (28 June 2006); doi: 10.1117/12.671982

Event: SPIE Astronomical Telescopes + Instrumentation, 2006, Orlando, Florida , United States 


\title{
Michigan Infrared Combiner (MIRC): Commissioning Results at the CHARA Array
}

\author{
John D. Monnier ${ }^{a}$, Ettore Pedretti $^{a}$, Nathalie Thureau ${ }^{a}$, Jean-Philippe Berger ${ }^{b}$, Rafael \\ Millan-Gabet $^{c}$, Theo ten Brummelaar ${ }^{d}$, Harold McAlister ${ }^{d}$, Judit Sturmann ${ }^{d}$, \\ Lazlo Sturmann $^{d}$, Phil Muirhead ${ }^{a, e}$, Ajay Tannirkulam $^{a}$, Scott Webster $^{a}$, and Ming Zhao ${ }^{a}$ \\ ${ }^{a}$ University of Michigan (Astronomy), 500 Church St, Ann Arbor, MI 48104 USA; \\ ${ }^{b}$ Lab. d'Astrophysique de l'Observatoire de Grenoble, Grenoble, France; \\ ${ }^{c}$ Michelson Science Center (Caltech), Pasadena, CA, USA; \\ ${ }^{d}$ Georgia State University CHARA Array, Mt. Wilson, CA, USA; \\ ${ }^{e}$ Now at Cornell University, Ithaca, NY, USA
}

\begin{abstract}
The Michigan Infrared Combiner (MIRC) has been designed for two primary goals: 1) imaging with all six CHARA telescopes simultaneously in the near-infrared, 2) direct detection of "hot Jupiter" exoplanets using precision closure phases. In September 2005, MIRC was commissioned on-sky at the CHARA Array on Mt. Wilson, CA, successfully combining light from 4 telescopes simultaneously. After a brief overview of MIRC features and design philosophy, we provide detailed description of key components and present results of laboratory tests. Lastly, we present first results from the commissioning run, focusing on engineering performance. We also present remarkable on-sky closure phase results from the first night of recorded data with the best-ever demonstrated closure phase stability and precision $(\Delta \Phi=0.03$ degrees $)$.
\end{abstract}

Keywords: imaging, interferometry, infrared, closure phase, infrared

\section{INTRODUCTION}

The Georgia State University (GSU) Center for High Angular Resolution Astronomy (CHARA) interferometer is a 6-telescope optical array located on Mt. Wilson, ${ }^{1}$ which recently published first science results ${ }^{2}$. In a collaboration with GSU, Dr. Monnier's group at the University of Michigan began in 2003 to build an instrument to act as an "imaging" infrared combiner to combine all six telescopes together to allow true interferometric imaging with CHARA from 1.45-2.5 $\mu \mathrm{m}$ with $\sim 1$ milliarcsecond resolution. The Michigan Infrared Combiner (MIRC) was first described in the last SPIE proceedings $;^{3}$ since that time, the MIRC combiner has been built, tested, and commissioned on the the sky.

\subsection{FEATURES OF MIRC COMBINER}

Here we briefly summarize the main properties of the MIRC combiner. Please refer to Monnier et al. ${ }^{3}$ for further details.

- Image Plane Combination: 6 telescopes, 15 visibilities, 20 closure phases simultaneously (and measurements of closure amplitudes for the first time); for commissioning, we used only 4 telescopes

- 1.45-2.5 microns ( $\mathrm{H}$ and $\mathrm{K}$ bands separately or simultaneously)

- Low-resolution spectroscopy, R 35, 150 or 450

- Spatial filtering using single-mode fibers

- Straight-forward integration with a separate fringe tracker (CHAMP, see Berger et al., these proceedings).

Further author information, J.D.M.: monnier@umich.edu

Advances in Stellar Interferometry, edited by John D. Monnier,

Markus Schöller, William C. Danchi, Proc. of SPIE Vol. 6268,

62681P, (2006) - 0277-786X/06/\$15 - doi: 10.1117/12.671982

Proc. of SPIE Vol. $626862681 \mathrm{P}-1$ 


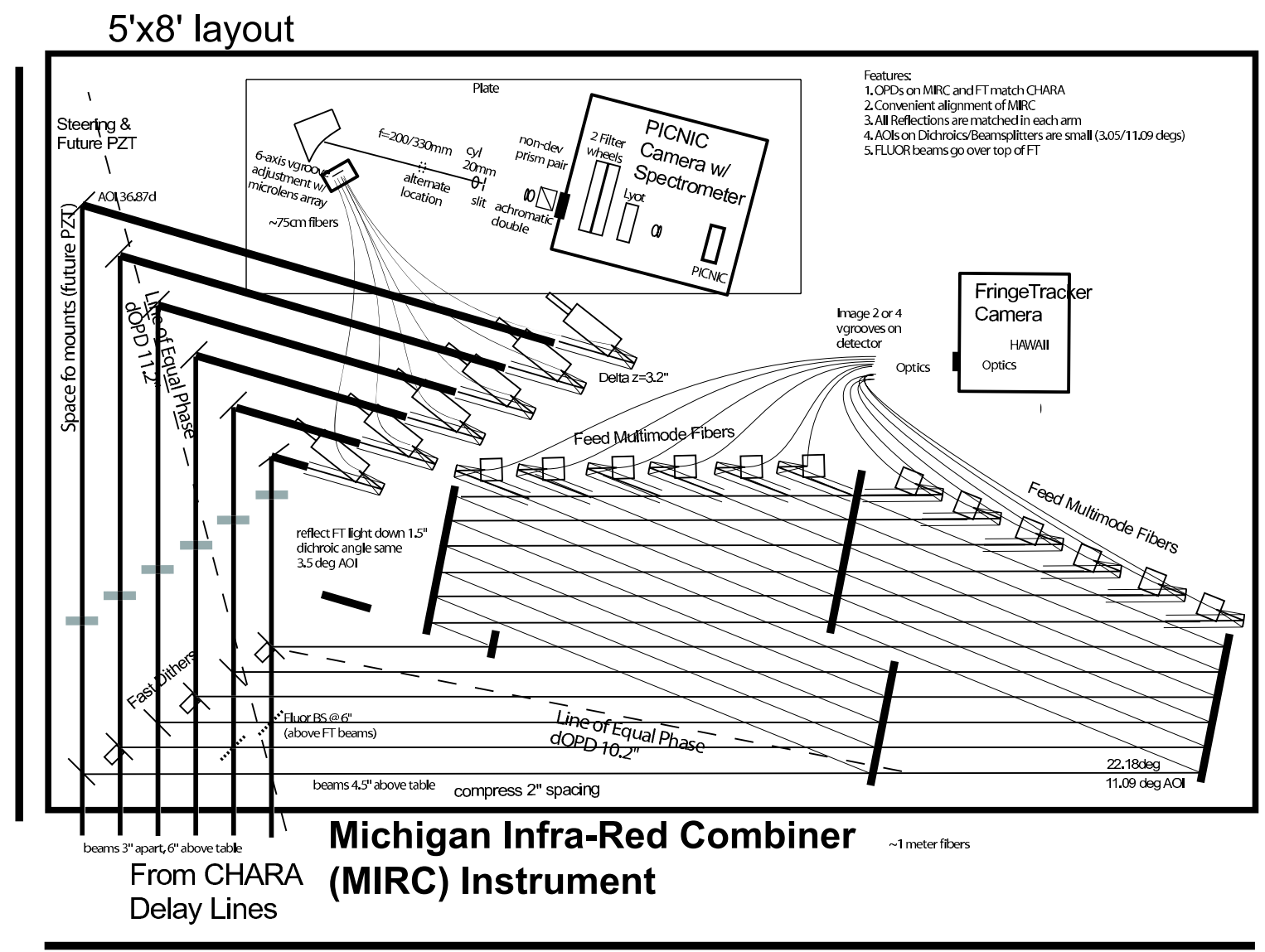

Figure 1. This figure shows the actual layout of the MIRC combiner (upper left part of optical table) as well as the planned location for the CHARA Fringe Tracker CHAMP (see paper by Berger et al. in these proceedings).

MIRC is an image-plane combiner, allowing simultaneous measurements of all available baselines and closure phases in the full 6-telescope CHARA array. The beams from all telescopes are arranged in a linear nonredundant pattern and then imaged onto the slit of an infrared spectrograph at the backend. In order to allow precision calibration of visibility amplitudes and closure phases, the synthetic pupil is formed using light transported and spatially-filtered by single-mode fibers for optical stability. ${ }^{4}$ A non-deviating prism pair $(\mathrm{R} \sim 35)$ or low-resolution grism $(\mathrm{R} \sim 150,450)$ on the output mitigates dispersion problems and bandwidth-smearing of data (especially important with the long CHARA baselines). This proposal is very similar to the recommended designs evaluated by Theo ten Brummelaar and Steve Ridgway in early CHARA technical memos, ${ }^{5,6}$ for example appendices $\mathrm{K}$ and $\mathrm{L}$.

The primary drawback to the above design is that this combiner will have relatively poor sensitivity, since both all-in-one beam combination and the dispersive element act to spread out the available light across many pixels (diluting the signal at the expense of additional read noise). In $\mathrm{K}$ band, there is another problem that in the synthetic pupil allows extra thermal background into the camera. However, these serious deficiencies can be overcome through future improved detectors and (in the short-term) use of a "fringe tracker."

We ascribe to the philosophy that mature interferometers will all have dedicated fringe tracking systems which act to correct for fast atmospheric fluctuations in realtime. In this way, a fringe tracker is like the now-common, adaptive optics systems found on most large telescopes. By separating out the light for fringe tracking from the light used for science, each fringe tracker and science combiner can be separately optimized. This is critical, since fringe trackers need lots of photons but good calibration is not needed. MIRC was designed with the assumption 
that an infrared fringe tracker would be built to recover the sensitivity lost ( $\sim 4$ magnitudes) in pursuing good calibration and powerful imaging capabilities - indeed, a fringe tracker will be integral to MIRC's ability to observe a wide-range of targets. The "CHARA-Michigan Phasetracker (CHAMP)" has now been funded by the National Science Foundation and will be built at the University of Michigan; our design is described elsewhere in these proceedings (Berger et al.).

This "science combiner with separate fringe tracker" design has been developed over many years, and was probably first best expressed by the CHARA team in their original NSF proposal. ${ }^{7}$ This represents a major departure from the approaches at the "first generation" imaging interferometers COAST and NPOI. We note that the European Southern Observatory Very Large Telescope Interferometer (ESO-VLTI) has adopted a similar architecture as we have for their AMBER instrument, ${ }^{8}$ which also will require a separate fringe tracker unit $\left(\right.$ FINITO $\left.^{9}\right)$ to achieve reasonable sensitivity at high spectral resolution.

Figure 1 shows how the MIRC combiner has been implemented at CHARA, and the next section will take you through a guided tour.
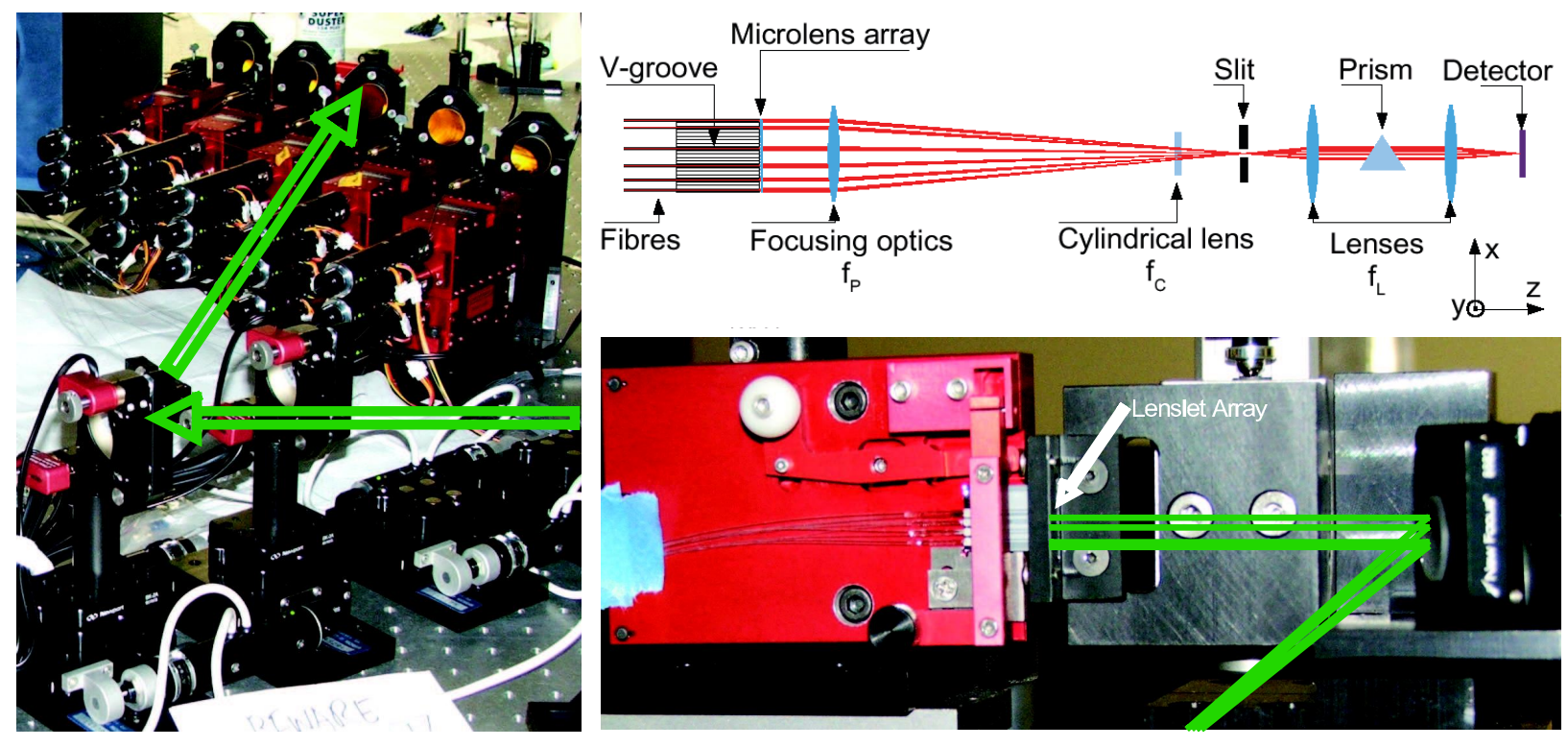

Figure 2. Here we begin our guided tour of the MIRC combiner. The left panel shows how the light is deflected out of the CHARA beampath into the MIRC instrument. You can see the off-axis parabolas that inject the light into single-mode fibers. Once in the fibers, the light takes the path shown in the upper-right schematic. The bottom right image shows the fibers being arranged into a silicon v-groove array, the location of the collimator lenslet array, and a spherical silver mirror that focuses the beams to produce interference fringes at the input slit of the camera-spectrometer.

\section{GUIDED TOUR}

\subsection{Forming Fringes}

After light is received by the 1-m CHARA telescopes, the beams are compressed to $19 \mathrm{~mm}$ and the sidereal delay is corrected by a combination of (in-vacuum) fixed delay segments and (in-air) dynamic delay line carts; see recent CHARA instrument paper ${ }^{1}$ for details. The six beams are split by a dichroic - the visible light is used for tip-tilt tracking using a new CCD-based system (see Sturmann et al., these proceedings), while the infrared light (JHK bands) is sent towards the MIRC combiner.

The 6 beams are picked-off by picomotor-steered mirrors on automated (Zabar/Ealing) translation stages (for phasing MIRC with internal CHARA internal fringes). Each $19 \mathrm{~mm}$ beam is injected into custom-drawn, low-OH silica fiber (Highwave; used by VLTI AMBER) using an EFFL 60mm off-axis parabola (gold-coated, 

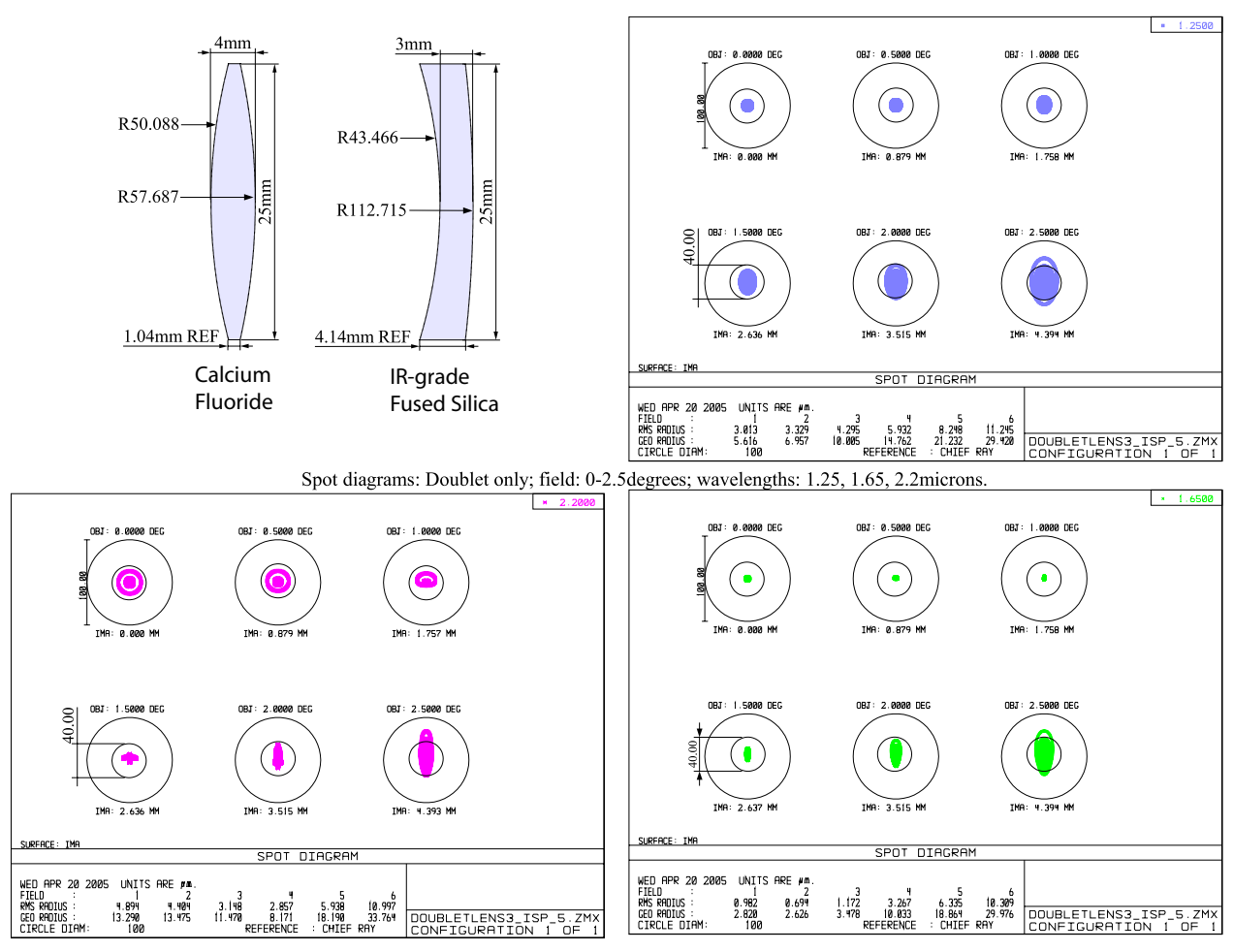

Figure 3. This figure shows the custom achromatic double designed for MIRC, requiring spot sizes of $<40 \mu \mathrm{m}$ over a 5 degree field from 1.1-2.4 $\mu \mathrm{m}$ (designed by N. Thureau). The upper left panel shows the design while the other panels show spot diagrams at $1.25 \mu \mathrm{m}, 1.65 \mu \mathrm{m}$, and $2.2 \mu \mathrm{m}$. We have verified that $\sim 90 \%$ of light does fall into a single $40 \mu \mathrm{m}$ row of pixels for $1.65 \mu \mathrm{m}$ and $2.2 \mu \mathrm{m}$ without re-focusing.

diamond-turned silicon mirrors from $\mathrm{Nu}$-Tek). Coupling is maximized by using 3-axis nanopositioning mounts (Luminos) to align fiber with $0.2 \mu \mathrm{m}$ repeatability (absolute) in $\mathrm{X}-\mathrm{Y}$ and a few microns of error in the focus direction.

The single-mode fibers $\left(\lambda_{\text {cutoff }}=1.45 \mu \mathrm{m}\right.$ ) are "highly-birefringent $(\mathrm{HB})$," or "polarization-maintaining (PM)," which means that linearly-polarized light injected along a fiber axis is maintained along the fiber path. In our case, we are using unpolarized light so all fibers have been aligned to a common orientation and care has been to taken to not stress the fibers. Stress will induce differential birefringence between the two polarization states, leading to reduced fringe contrast in unpolarized light. We have provision for placing an in-line, squeeze-style polarization controller (General Photonics) on each fiber to correct for birefringence, however we found only 1 fiber needed this correction.

All 6 fibers are then arranged on a silicon v-groove array (the fiber array was populated and packaged by $\mathrm{Oz}$ Optics) with an exact $250 \mu \mathrm{m}$ pitch. The fibers are arranged in a non-redundant spacing so that each fringe that forms will have a unique spatial frequency signature. Light exiting a fiber is strongly diverging until collimated by a microlens array (SUSS MicroOptics) placed $\sim 100 \mu \mathrm{m}$ from the tip of the v-groove. The collimated beams form a synthetic pupil which is imaged using a $\mathrm{R}=400 \mathrm{~mm}$ (for 4 beams) or $750 \mathrm{~mm}$ (for 6 beams) focusing mirror (Lambda Research Optics).

All of these steps are illustrated in Figure 2.

\subsection{MIRC Spectrograph}

Since the fringes are forming in only one dimension, it is advantageous to compress the fringe pattern into a line of pixels. We use a $\mathrm{f}=20 \mathrm{~mm}$ cylindrical lens $\left(\mathrm{CaF}_{2}\right.$, ISP Optics) to do this for 4 beams $(\mathrm{f}=30 \mathrm{~mm}$ will be needed 

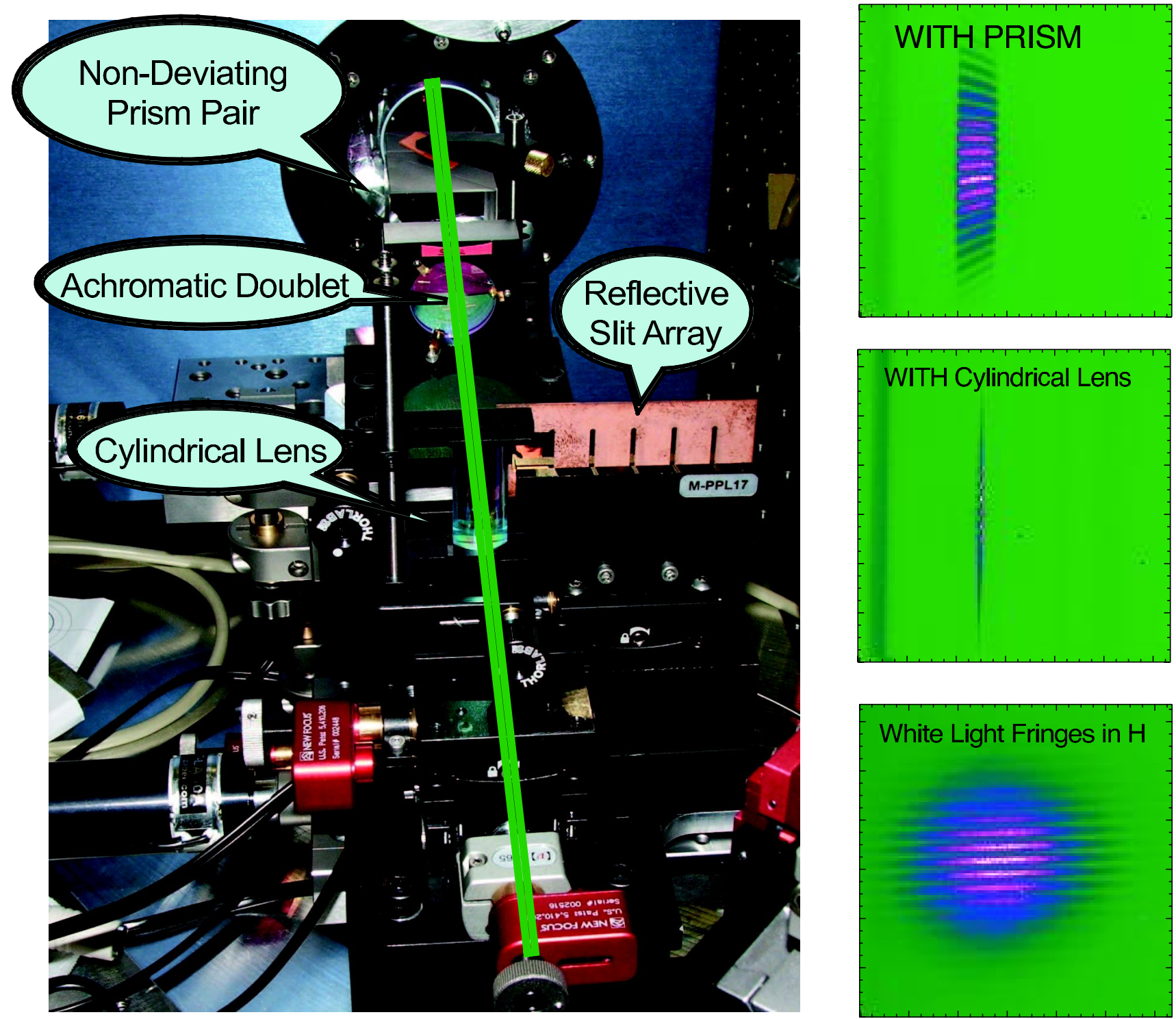

Figure 4. This figure shows the transformation of the fringes through the MIRC spectrograph. The combined light from all the fibers arrives from the bottom of the left-hand figure. The fringes are focused onto the reflective slit array, and the bottom-right image shows laboratory fringes from two fibers without the cylindrical lens. In order to disperse fringes we insert a cylindrical lens to squash the light into a single line of pixels on the detector (see middle-right panel). Next, this line of fringes is sent through the spectrograph, consisting of a custom achromatic doublet (collimator), a non-deviating prism pair, and then into the dewar where the light is further bandpass-filtered and re-imaged onto the detector (see upper-right, where wavelength varies along x-axis and the fringes are apparent on the y-axis). We also have two grisms inside the dewar which can be used instead of the prism pair (see Figure 5). 

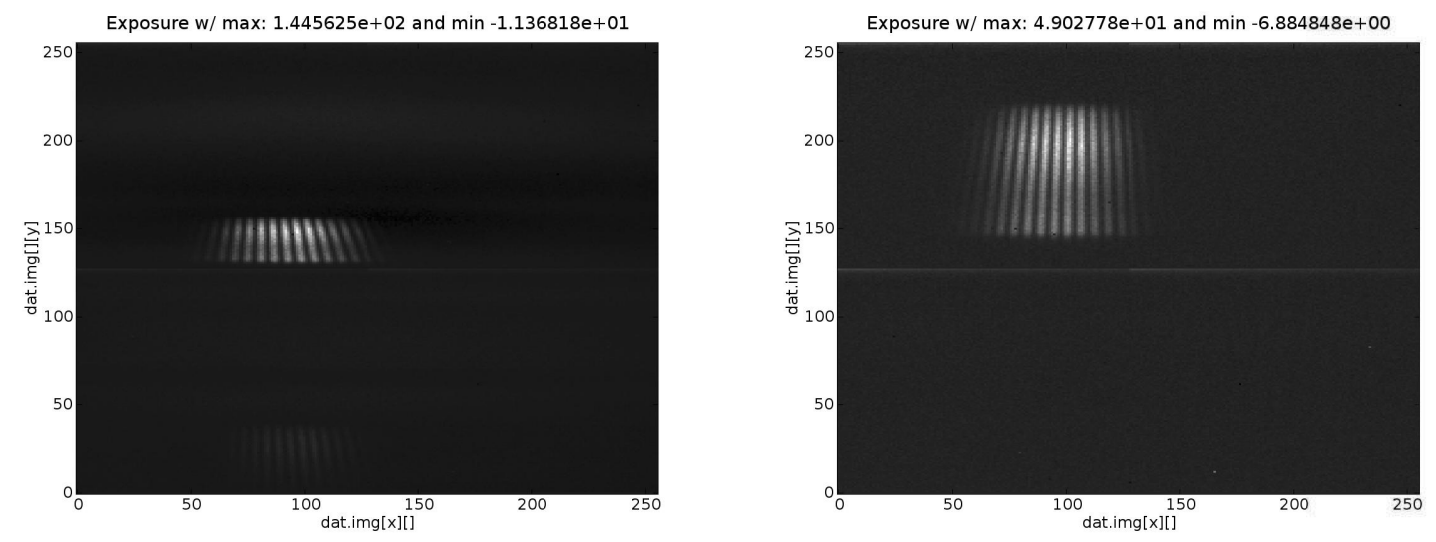

Figure 5. Here we present first (lab) fringes across $\mathrm{H}$ band using the low $(\mathrm{R}=150)$ and high $(\mathrm{R}=450)$ resolution grisms inside the MIRC dewar (wavelength along y axis and fringes along $\mathrm{x}$ axis). The grisms work out to $\mathrm{K}$ band but suffer from increased thermal background since the input slit is not cooled. Note there is neglible differential dispersion in these fringes, indicating the fibers are nearly equal in length $(\Delta x<0.25 \mathrm{~mm})$.

for 6 beams). The fringes come to a line-focus onto a reflective slit array (gold-foiled; National Aperture), which defines the entrance aperture of the spectrograph. The gold foil acts to reduce the infrared background entering the camera for K-band operation - ideally, the slit would be cryogenic and located in the cold volume of the camera, but this upgrade will drastically increase alignment difficulties and has been deferred.

The beam expands from the slit and is collimated by a $\mathrm{f}=100 \mathrm{~mm}$ custom acromatic doublet (design by N. Thureau, fabricated by ISP Optics; see Figure 3). The collimated beam is then dispersed either using a nondeviating prism pair (design by W. Traub, fabricated by Red Optronics \& Planar Optics) or infrared grisms. At this point, the light enters the MIRC camera dewar through an Infrasil window (Esco). The $\mathrm{LN}_{2}$ dewar was made by IR Labs and has two automated filter wheels and a hold time 48 hours. After passing through a bandpass filter, the light is re-imaged onto a $256 \times 256$ pixel PICNIC (Rockwell) detector.

Figure 4 shows both a picture of the spectrograph as well as recorded images of interference fringes with and without the cylindrical lens in place.

In April 2006, we installed custom grisms (Newport/Spectra Physics) into the MIRC dewar, in one of the filter wheels. This allows us to observe with 2 additional levels of spectral resolution $(\mathrm{R}=150$ and 450$)$. While these two grisms have not been used on the sky, we present validation fringes in Figure 5 using a laboratory white light source.

As mentioned, the detector is a Rockwell PICNIC array detector, sensitive from 1 to $2.5 \mu \mathrm{m}$. This combiner requires reading-out a relatively large area $(\sim 50 \times 120$ pixels $)$ at high rates $(\gtrsim 20 \mathrm{~Hz})$ with lowest possible readnoise. We are using the cold fanout board and warm analog amplifiers from IR Labs and using high-speed digital electronics (including ADCs) from Astronomical Research Cameras (Bob Leach). We have demonstrated $23 \mathrm{e}^{-}$ read noise for single reads at $1 \mathrm{MHz}$ pixel rates, comparable to that achieved at IOTA $^{10}$ after correcting for our faster pixel clock. Custom DSP timing patterns have been written to maximize signal-to-noise ratio (by optimizing use of non-destructive reads and Fowler sampling; Leach \& Monnier) and to allow sophisticated data analysis, such as employing variable coherent integration times in post-processing.

In order to have full control of the data acquisition and realtime feedback to the CHARA interferometer for fringe tracking, the camera has been interfaced under linux with the RTAI-FUSION realtime extension (see Pedretti et al, these proceedings). Using the fastest fiber interface board offered by Leach (12.5 Mpixels/sec), we can readout the PICNIC array at maximum data rates, retaining every non-destructive read and spooling to disk with no latency or loss of integration time (see Pedretti et al, these proceedings for details). With 4 

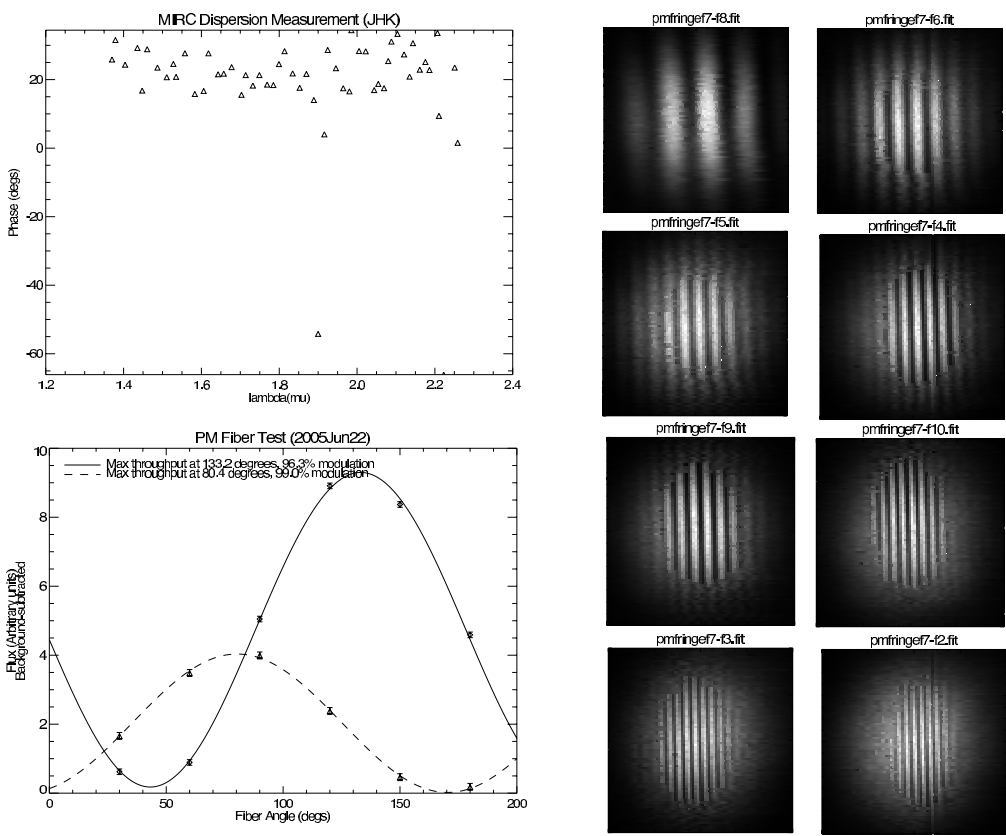

Figure 6. This figure shows the results of 3 laboratory tests of the MIRC combiner. The upper-left panel shows there was neglible differential dispersion between the two fibers under test. The bottom-left panel shows the results of the polarization alignment test. The nearly $100 \%$ modulation shown here indicates that there is neglible cross-talk between the fast and slow guided modes in the fiber (and that Oz Optics did a good job aligning the PM fibers in the v-groove array). The right panel shows 8 fringe patterns made between fibers in the silicon v-groove array using the broadband $\mathrm{H}$ filter (for this experiment, no cylindrical lens was used). The fringe frequency increases from top to bottom, corresponding to increasing fiber spacings in the v-groove pattern. We were able to demonstrate typically $90 \%$ (broadband $\mathrm{H}$ ) fringe visibilities between fiber pairs in unpolarized light.

readout amplifiers going at full speed, this produces data at the impressive rate of 8 Megabytes per second, or 288 Gigabytes for 10 hours of integration!

It is worth noting that all aspects of the MIRC instrument are remote controlled. We plan to implement automatic alignment procedures in the future to allow the broad CHARA observing community to use MIRC without requiring specialized training.

\subsection{Laboratory tests}

Many of the technologies used in this combiner have never been used for astronomical purposes. Figure 6 shows the results of rigorous laboratory tests that confirmed critical performance for this novel combiner before shipping to Mt. Wilson. The tests confirmed that we were able to equalize the fiber lengths to within $0.25 \mathrm{~mm}$ (or better) following the procedure of Burnett \& Jones ${ }^{11}$ - proven by the almost complete lack of any observed differential dispersion seen in the fringes across $\mathrm{J}, \mathrm{H}$, and $\mathrm{K}$ bands.

The lab tests also showed there was minimal cross-talk between the fast and slow guided modes of the PM fiber. To test this, linearly polarized light was injected into one fiber and an analyzer was placed at the output of the v-groove before detection by the infrared camera. By rotating the input fiber we can align all the fibers to a common axis. Lastly, we showed that high contrast fringes $(V \sim 90 \%)$ were possible with all the fibers using unpolarized light and an in-line, squeeze-style polarization controller (General Photonics). These tests gave us great confidence before attempting on-sky tests at CHARA. 


\section{ON-SKY RESULTS}

Commissioning of the MIRC instrument was carried out from UT August 26 to UT September 19, 2005. We planned on commissioning only 4 of the 6 CHARA telescopes, since two beams are currently devoted to other projects. First fringes with MIRC on the sky were obtained on UT 2005 Sep 04 and first closure phases with CHARA on 2005 Sep 14. The most ambitious of our technical goals was to combine light from all four telescopes simultaneously, and this was achieved first on UT 2005 Sep 18 and again on the subsequent night.

One of the critical reasons for our successes was the sophisticated graphical user interfaces (GUIs) programmed using python pyGTK that we were developed during the commissioning run in response to unexpected needs. Thureau et al. (these proceedings) give more details about the benefits of using TCP/IP sockets and python for creating flexible, "lightweight," and attractive GUI interfaces which were critically needed for controlling the instrument and searching multiple delay lines for fringes.

Figure 8 shows the results from our first 4-telescope observing. The fringes from all baselines are detected and real-time fringe tracking was implemented during observing. This commissioning run did not result in any astronomically-useful data since we achieved this milestone on the penultimate night of our run. The first science runs are planned for June and August of 2006.

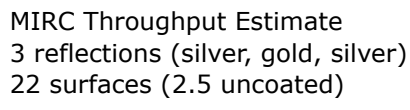

$\mathrm{H}$

$\mathrm{K}$

Silver Pickoff
Gold OAP
Fiber Coupling (5cm r0)
Fiber transmission (1m)
Fiber Fresnel
Lenslet transmission
Lenslet Fresnsel
Mirror (silver)
Cylindrical (AR)
Slit
Doublet (AR)
Prism Pair (AR)
Window (AR)
Bandpass Filter
Doublet (AR)
Detector (QE)
Expected Transmission

$\begin{array}{ll}98 \% & 98 \% \\ 98 \% & 98 \% \\ 25 \% & 36 \% \\ 95 \% & 90 \% \\ 92 \% & 92 \% \\ 99 \% & 90 \% \\ 97 \% & 92 \% \\ 98 \% & 98 \% \\ 97 \% & 97 \% \\ 95 \% & 95 \% \\ 94 \% & 94 \% \\ 92 \% & 92 \% \\ 97 \% & 97 \% \\ 85 \% & 85 \% \\ 94 \% & 94 \% \\ 60 \% & 60 \% \\ 7.3 \% & 8.6 \%\end{array}$

Figure 7. Here we show our accounting for the expected throughput of MIRC. Care was taken to include broadband AR coating on most optics and most of the loss is due to coupling into fibers since the CHARA telescopes are larger than the Fried coherence length $r_{O}$ in the near-infrared.

We were able to track fringes on a star as faint as $\mathrm{H}=3.0$ ( $v$ And) under poor seeing conditions. The net throughput of CHARA+MIRC during this run (at $1.65 \mu \mathrm{m}$ ) was a dissappointing $0.27 \%$ (including all losses including detector $\mathrm{QE}$ ), while we were expecting $2.5 \%$. We believe that poor beam image quality was partly to blame for our low observed throughput, since this drastically hurts the coupling into the single-mode fibers. Other CHARA combiners show 3-6× lower throughput than expected, suggesting some of the throughput loss is likely unrelated to the MIRC instrument. Increasing throughput is a primary goal of CHARA engineering this year, and more on-sky experience will allow a thorough characterization of the MIRC throughput in $\mathrm{H}$ and $\mathrm{K}$ bands.

The main scientific result from the MIRC commissioning is presented in Figure 9 where we analyzed approximately 1.5 hours of closure phase data on $\beta$ Tau. Recall that one of the primary design goals of MIRC was to have good stability of the closure phase in order to carry out "precision closure phase" studies of extrasolar planets. ${ }^{12}$ 

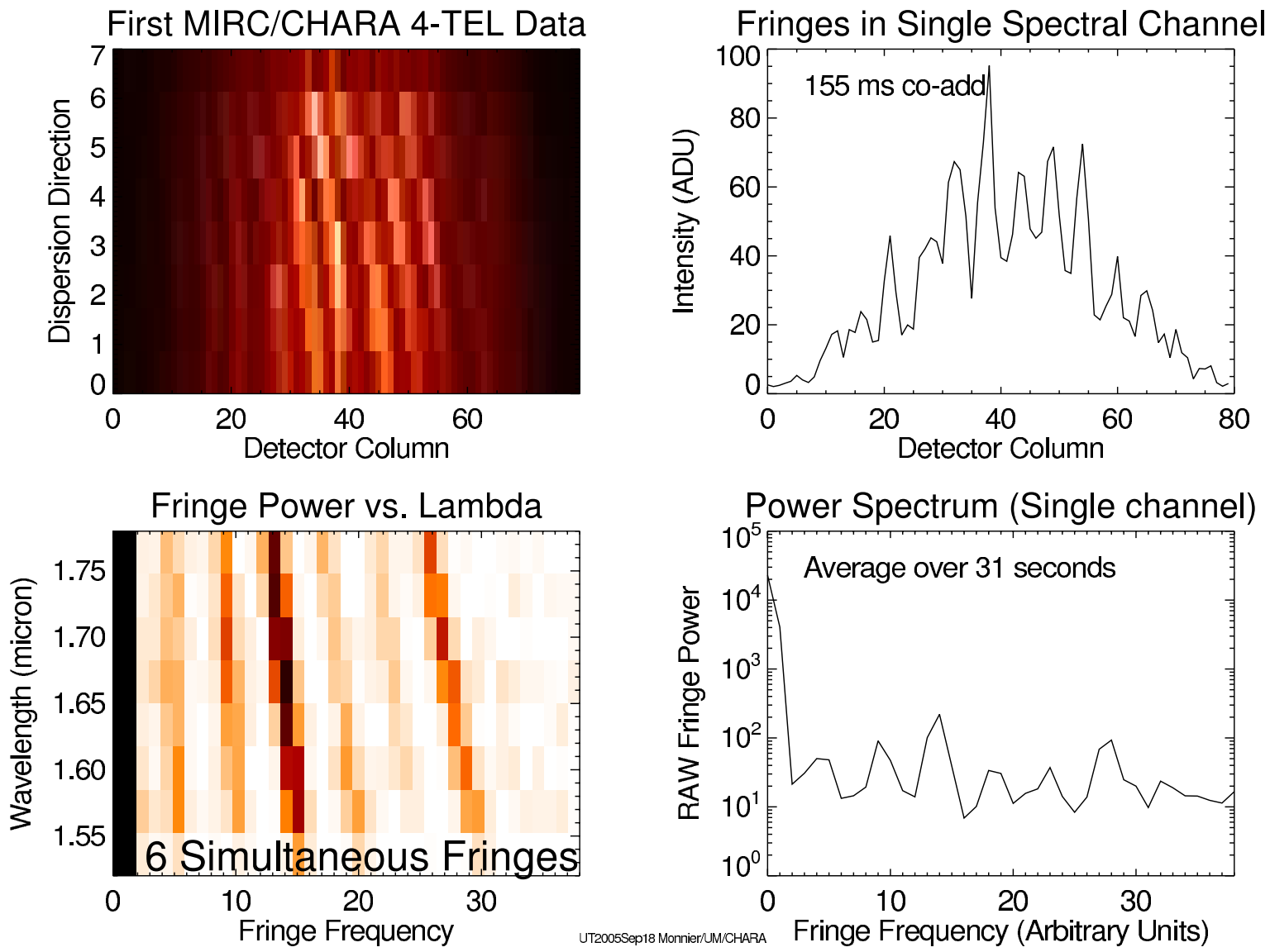

Figure 8. This figure documents the first 4-telescope fringes at CHARA using MIRC. The top-left panel shows a snapsot of the dispersed fringes - although difficult to see, there are 6 different fringes in this pattern. The top-right shows a scan through one of the wavelength channels to show the superimposed fringes patterns. The bottom-left panel shows the line-by-line powerspectrum of the fringes, averaged over one data file ( $\sim 31 \mathrm{sec})$ - note how each of the 6 fringes are well-separated in Fourier-space, eliminated cross-talk effects which continue to plague time-domain all-in-one combiners. The bottom-right shows the power spectrum of a single channel to give a more quantitative picture of the data quality. Note that the target was the well-known binary $\iota \mathrm{Peg}$ and some visibility variation with baseline is expected. 

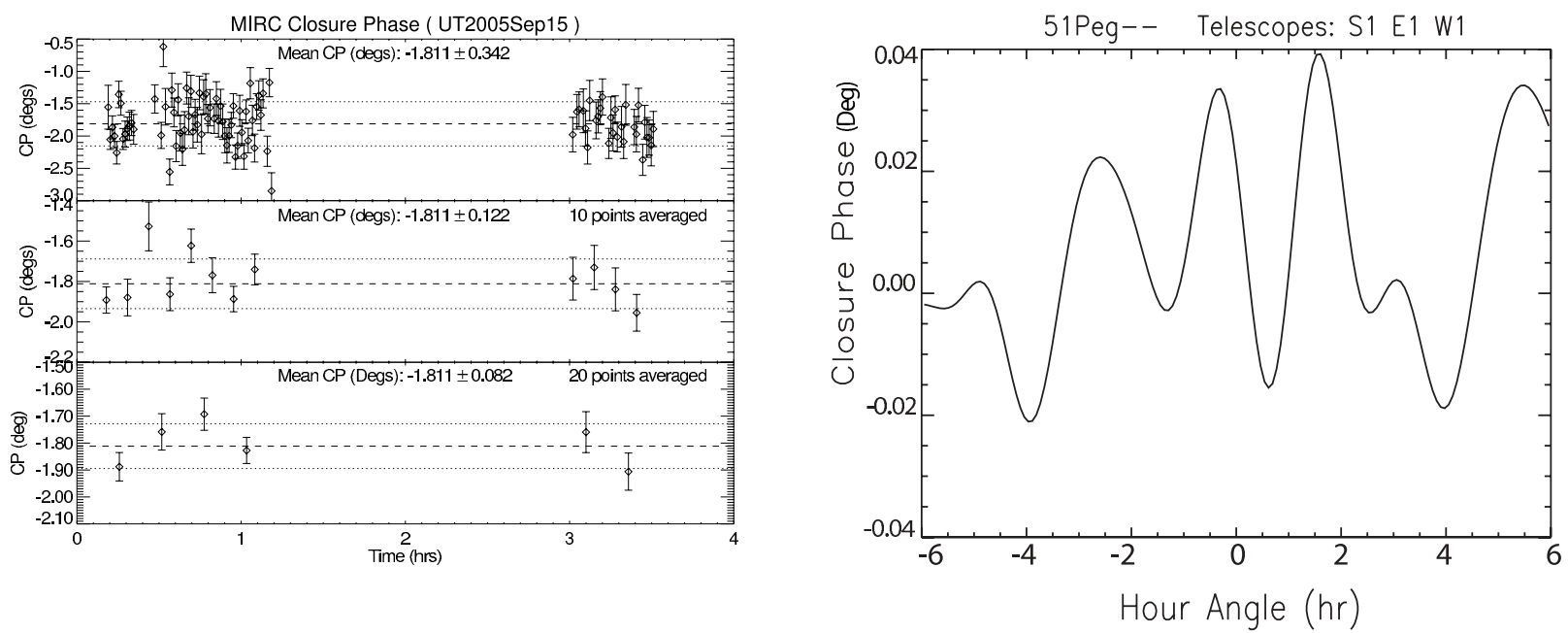

Figure 9. The left figure shows the results of a closure phase study of Beta Tau (H=1.9) from UT2005Sep15. The top panel shows the closure phase results (averaged over all wavelength channels in $\mathrm{H}$ band) for each data file. The middle panel shows the result of averaging 10 files, and the bottom averages 20 files. Note that the error reduces like $\sqrt{N}$, indicating the measurement is immune from "systematic" effects which typically corrupt the visibility measurements due to changes in the seeing. Furthermore, there are no apparent drifts in the calibration over period of 3 hours. The formal error on the closure phase for the entire night is $\Delta \Phi \sim 0.03$ degs, approximately the size of the maximum closure phase for the hot Jupiter around $51 \mathrm{Peg}$ (see right figure). This initial measurement suggests that Hot Jupiters will be detectable at CHARA with MIRC using closure phases; a main challenge will be to increase system throughput to overcome photon noise.

In the very first night of recorded closure phases, we see here that the closure was stable within measurement errors over a period of 3 hours. This is about an order of magnitude more stable than other combiners at IOTA and NPOI. Furthermore, we demonstrate that the closure phase error averages down like $\sqrt{N}$, indicating that systematic errors do not play a major role in our measurement.

The encouraging result is that we have achieved a closure phase measurement with a formal error (on the mean) of $\Delta \Phi \sim 0.03$ degrees, which is the best measurement ever documented for closure phase with an optical interferometer. Excitingly, we see in the right panel of Figure 9 that this error level is close to the expected signal from the "hot Jupiter" planet around 51 Peg. We will continue to develop the precision closure phase method for direct extrasolar planet detection, however we will need greater throughput (and/or the planned fringe tracker upgrade) before we will be successful in detecting an extrasolar planet. Of course, further tests will also be needed before being confident that we have indeed overcome all the major systematic errors.

\section{ACKNOWLEDGMENTS}

We gratefully acknowledge funding from the University of Michigan for construction and commissioning of the MIRC combiner. The design concept is the result of conversations with many interferometrists, and the authors have appreciated their input. We have tried to take advantage of lessons learned in the fabrication and use of recent combiners, in particular from NPOI, COAST, AMBER (VLTI) and FATCAT (Keck Interferometer). We specially thank G. Vasisht and G. Perrin for specific help with regard to IR camera and fiber issues. We also acknowledge the important help of Guangyu Li who helped polish the v-groove array. E. Pedretti acknowledges support from a NASA Michelson Fellowship and N. Thureau was supported by a Marie Curie International Outgoing Fellowship. Thank you to P.J. Goldfinger, Nils Turner, Bob Cadman, Steve Golden and Mandy PaigeHenderson for technical and/or observing support at CHARA during commissioning - we always miss your emails after leaving. Lastly, we acknowledge the State of Georgia, the National Science Foundation, the W.M. Keck Foundation, and the Lucille and David Packard Foundation for funding the construction of the CHARA array; array operations are being supported by Georgia State University and NSF-AST 0307562. 


\section{REFERENCES}

1. T. A. ten Brummelaar, H. A. McAlister, S. T. Ridgway, W. G. Bagnuolo, N. H. Turner, L. Sturmann, J. Sturmann, D. H. Berger, C. E. Ogden, R. Cadman, W. I. Hartkopf, C. H. Hopper, and M. A. Shure, "First Results from the CHARA Array. II. A Description of the Instrument," ApJ 628, pp. 453-465, July 2005.

2. H. A. McAlister, T. A. ten Brummelaar, D. R. Gies, W. Huang, W. G. Bagnuolo, M. A. Shure, J. Sturmann, L. Sturmann, N. H. Turner, S. F. Taylor, D. H. Berger, E. K. Baines, E. Grundstrom, C. Ogden, S. T. Ridgway, and G. van Belle, "First Results from the CHARA Array. I. An Interferometric and Spectroscopic Study of the Fast Rotator $\alpha$ Leonis (Regulus)," ApJ 628, pp. 439-452, July 2005.

3. J. D. Monnier, J.-P. Berger, R. Millan-Gabet, and T. A. Ten Brummelaar, "The Michigan Infrared Combiner (MIRC): IR imaging with the CHARA Array," in New Frontiers in Stellar Interferometry, Proceedings of SPIE Volume 5491. Edited by Wesley A. Traub. Bellingham, WA: The International Society for Optical Engineering, 2004., p.1370, W. A. Traub, ed., pp. 1370-+, Oct. 2004.

4. V. Coudé du Foresto, S. Ridgway, and J.-M. Mariotti, "Deriving object visibilities from interferograms obtained with a fiber stellar interferometer," A\&AS 121, pp. 379-392, Feb. 1997.

5. T. ten Brummelaar, "Visible light imaging," Tech. Rep. Appendix-K, CHARA Array, Georgia State University, Atlanta, GA, 1993.

6. S. Ridgway, "Infrared beam combining and detection," Tech. Rep. Appendix-L, CHARA Array, Georgia State University, Atlanta, GA, 1993.

7. T. ten Brummelaar, "Visible light imaging," Tech. Rep. Appendix-I, CHARA Array, Georgia State University, Atlanta, GA, 1993.

8. F. Malbet, R. G. Petrov, I. Tallon-Bosc, K. Hofmann, D. Mourard, T. Forveille, F. Cassaing, F. Reynaud, M. Tallon, P. Mege, and K. Rousselet-Perraut, "System analysis of the AMBER instrument on VLTI," in Interferometry for Optical Astronomy II. Edited by Wesley A. Traub . Proceedings of the SPIE, Volume 4838, pp. 1163-1170 (2003)., pp. 1163-1170, Feb. 2003.

9. M. Gai, L. Corcione, L. Delatge, D. Gardiol, A. Gennai, M. G. Lattanzi, D. Loreggia, G. Massone, S. Menardi, and F. Reynaud, "The FINITO Fringe Sensor for VLTI," in Beyond conventional adaptive optics. ESO Conference and Workshop Proceedings, Vol. 58, ISBN 3923524617, pp. 329-+, 2002.

10. E. Pedretti, R. Millan-Gabet, J. D. Monnier, W. A. Traub, N. P. Carleton, J.-P. Berger, M. G. Lacasse, F. P. Schloerb, and M. K. Brewer, "The PICNIC Interferometry Camera at IOTA," PASP 116, pp. 377-389, Apr. 2004.

11. J. G. Burnett and J. D. C. Jones, "Cutting optical fibers to equal lengths for broadband stellar interferometry," Appl. Opt. 31, pp. 2977-+, June 1992.

12. J.-L. Beuzit, D. Mouillet, B. R. Oppenheimer, and J. D. Monnier, "Direct detection of exoplanets," in Protostars and Planets V, 2006. 